\title{
THE BOHR SPECTRUM OF A BOUNDED FUNCTION
}

\section{H. G. EGGLESTON}

C. S. Herz, [2], has conjectured the following result.

If $\phi(x)$ is a bounded uniformly continuous function of the real variable $x$, then for almost all real values of $t$,

$$
\lim _{N \rightarrow \infty} \frac{1}{2 N} \int_{-N}^{N} \exp (-i t x) \phi(x) d x=0 .
$$

In this note it is shown that this conjecture is correct. The exceptional set may however be non-enumerable. The result is a consequence of a connection between the set of values $t$ for which (1) is false and that for which the nonintegral parts of the sequence $\left\{n_{k} t\right\}$ are not equidistributed, where $n_{k}$ is an increasing sequence of integers satisfying $n_{k+1}-n_{k}<C$ for some constant $C$.

Definitions and Notation. The set of values of $t$ for which (1) is false will be denoted by $T(\phi)$ and any set which is a $T(\phi)$ set for a bounded and uniformly continuous function $\phi(x)$ will be said to be of type I.

The set of values of $t$ for which the nonintegral parts of the sequence $\left\{n_{k} t\right\}$ are not equidistributed will be denoted by $T\left\{n_{k}\right\}$. If the sequence $\left\{n_{k}\right\}$ is a strictly increasing sequence of positive integers for which $n_{k+1}-n_{k}<C$ where $C$ is a constant, then the set $T\left\{n_{k}\right\}$ is said to be of type II.

$\Gamma$ will be used to denote an outer measure function in the sense of Carathéodory.

If $l$ is a real number, $[l]$ denotes the largest integer not greater than $l$.

The connection between equidistribution and the property expressed in (1) is given by the following theorem

TheOREM. (a) If the $\Gamma$ measure of every set of type II is zero then so is the $\Gamma$ measure of every set of type $\mathrm{I}$.

(b) If the $\Gamma$ measure of every set of type I is zero then so is the $\Gamma$ measure of every set of type II.

Proof of (a). Suppose that, in contradiction to the statement of (a), there exists a set $T$ of type I such that $\Gamma(T)>0$, even though the $\Gamma$ measure of every set of type II is zero. Then for the appropriate bounded and uniformly continuous function $\phi(x)$

Received by the editors July 17, 1957 and, in revised form, September 13, 1957. 


$$
\limsup _{N \rightarrow \infty} \frac{1}{2 N}\left|\int_{-N}^{N} \exp (-i t x) \phi(x) d x\right|>0 \quad t \in T,
$$

and hence there is a positive number $\eta$ and a subset $T_{1}$ of $T$ such that $\Gamma\left(T_{1}\right)>0$ and

$$
\limsup _{N \rightarrow \infty} \frac{1}{2 N}\left|\int_{-N}^{N} \exp (-i t x) \phi(x) d x\right|>\eta \quad t \in T_{1} .
$$

The integral is written as the sum of eight integrals obtained by considering separately, the positive and negative ranges of $x$, the real and imaginary parts of exp $(-i t x)$, the real and imaginary parts of $\phi(x)$. Of these eight integrals there is at least one for which the corresponding upper limit is greater than $\eta / 8$ for all $t$ of a subset $T_{2}$ of $T_{1}$ where $\Gamma\left(T_{2}\right)>0$. Suppose for definiteness that $\psi(x)$ is the real part of $\phi(x)$ and that

$$
\limsup _{N \rightarrow \infty} \frac{1}{N}\left|\int_{0}^{N} \psi(x) \cos t x \cdot d x\right|>\frac{1}{4} \eta \quad t \in T_{2} .
$$

The argument in the other cases is similar.

Since $\phi(x)$ is uniformly continuous the same is true of $\psi(x)$ and thus there is a positive number $\delta$ such that

$$
|\psi(x)-\psi(y)|<\frac{1}{8} \eta \quad|x-y|<\delta .
$$

Let $M$ be a positive integer such that $M>8 / \eta$ and define $\chi(x)$ by

$$
\chi(x)=\sup _{m \delta \leqq y<(m+1) \delta}[\psi(y) M] / M, \quad m \delta \leqq x<(m+1) \delta, m=0,1, \cdots
$$

Then $\chi(x)$ is constant throughout the intervals $m \delta \leqq x<(m+1) \delta$ and if we denote its value in this interval by $a_{m}$ this implies (writing $J$ for $[N / \delta]-1)$,

$$
\limsup _{J \rightarrow \infty} \frac{1}{J}\left|\sum_{0}^{J} a_{m}(\sin m t \delta-\sin (m+1) t \delta)\right|>\frac{1}{8} \eta \delta|t| \quad t \in T_{2} .
$$

But the $a_{m}$ are rational numbers of the form $p / M$ and they take at most $[2 \sup |\psi(x)|+1] M=K$ different values. Let $\left\{b_{m, p}\right\}$ be the sequence defined by

$$
\begin{array}{rlr}
b_{m, p} & =a_{m} & \text { for those } m \text { for which } a_{m}=p / M \\
& =0 & \text { for all other } m .
\end{array}
$$

There are $K$ such sequences $\left\{b_{m, p}\right\}$ corresponding to the different pos- 
sible values of $p$ and the sequence $\left\{a_{m}\right\}$ is the sum of these $K$ sequences. It is easy to deduce that there exists a sequence of integers $\left\{m_{k}\right\}$ such that $m_{k+1}>m_{k}, m_{k+1}-m_{k}<C$ where $C$ is an appropriate constant, and

$$
\limsup _{J \rightarrow \infty} \frac{1}{J}\left|\sum_{m_{k} \leqq J}\left(\sin m_{k} t \delta-\sin \left(m_{k}+1\right) t \delta\right)\right|>0, \quad t \in T_{4}
$$

where $\Gamma\left(T_{4}\right)>0$. But $\Gamma\left(T\left\{m_{k}\right\}\right)=0$ and thus there is a $t \in T_{4}$ such that $t \delta \notin T\left\{m_{k}\right\}$. Hence

$$
\lim _{K \rightarrow \infty} \frac{1}{K} \sum_{K=1}^{K} \sin \left(m_{k} t \delta\right)=0 .
$$

Since this implies that

$$
\underset{J \rightarrow \infty}{\limsup } \frac{1}{J}\left|\sum_{m_{k} \leqq J}\left(\sin m_{k} t \delta-\sin \left(m_{k}+1\right) t \delta\right)\right|=0
$$

we have a contradiction with (4) and this establishes the correctness of (a) of the theorem.

Proof of (b). Let $T$ be a set of type II and suppose that $\Gamma(T)>0$ even though the $\Gamma$ measure of every set of type $I$ is zero. Then there is a sequence $\left\{n_{k}\right\}$ and a constant $C$ such that $n_{k+1}-n_{k}<C$ and for every $t \in T$ the nonintegral parts of $\left\{n_{k} t\right\}$ are not equidistributed. By [3] this means that for every $t \in T$ there is an integer $h$ such that

$$
\limsup _{K \rightarrow \infty} \frac{1}{K}\left|\sum_{k=1}^{K} \exp \left(-i n_{k} h t\right)\right|>0 .
$$

Since $\Gamma(T)>0$ there is a subset of $T, T_{1}$ for which (5) is true for a fixed integer $h$ and further $\Gamma\left(T_{1}\right)>0$. There is a positive number $\eta$ and a subset $T_{2}$ of $T_{1}$ such that

$$
\limsup _{K \rightarrow \infty} \frac{1}{K}\left|\sum_{k=1}^{K} \exp \left(-i n_{k} h t\right)\right|>\eta, \quad t \in T_{2}, \Gamma\left(T_{2}\right)>0 .
$$

There is a subset $T_{3}$ of $T_{2}$ such that $\Gamma\left(T_{3}\right)>0$ and, for some positive number $\delta$

$$
\frac{1}{3(C+1) h}\left|\frac{\sin t}{t}\right|>\delta \quad t \in T_{3} .
$$

Define the function $f_{k}(x)$ by 


$$
\begin{array}{rr}
f_{k}(x)=1 & n_{k} h-1 \leqq x<n_{k} h+1, k=1,2, \cdots, \\
& =0
\end{array}
$$

Define the function $\psi(x)$ by

$$
\psi(x)=\sum_{k=1}^{\infty} f_{k}(x)
$$

and let the function $\phi(x)$ be a uniformly continuous function such that in each interval $m \leqq x \leqq m+1, \phi(x)=\psi(x)$ except possibly for $x$ lying in two subintervals whose total length is at most $\eta \delta / 2$ and then for all $x$ it can be seen that

$$
\limsup _{N \rightarrow \infty} \frac{1}{2 N}\left|\int_{-N}^{N} \exp (-i t x) \phi(x) d x\right| \geqq \frac{1}{2} \eta \delta \quad t \in T_{3} .
$$

Thus $T_{3}$ is a subset of a set of type I and we have a contradiction since $\Gamma\left(T_{3}\right)>0$ whereas $\Gamma(T)=0$ for every set $T$ of type I.

This completes the proof of (b).

REMARKS (1). Herz's conjecture is an immediate consequence of Weyl's theorem in [3] that $\left\{n_{k} t\right\}$ is equidistributed for almost all $t$. It has recently been shown by P. Erdös and S. J. Taylor [1] that the set of $t$ for which $\left\{n_{k} t\right\}$ is not equidistributed has measure zero with respect to the Hausdorff measure defined by $(\log (1 / t))^{-1-\epsilon}, \epsilon>0$ provided $n_{k+1}-n_{k}<C$ for a constant $C$. Thus the exceptional set $t$ in (1) also has zero measure with respect to this measure function. In particular it is of zero fractional dimension.

(2) The connection between the two types of exceptional sets can be made even closer. If for some outer measure function $\Gamma$ the sup. of $\Gamma(T)$ where $T$ is of type $I$ is finite say $F$ then this number is also the sup. of $\Gamma(T), T$ of type II. The argument is similar to that used above with the observation that if two sets $T_{1}$ and $T_{2}$ are subsets of $T\left\{n_{k}^{(1)}\right\}$ and $T\left\{n_{k}^{(2)}\right\}$ respectively then we can find a third sequence $\left\{n_{k}^{(3)}\right\}$ such that all of $T_{1} \cup T_{2}$ except a subset of arbitrarily small $\Gamma$ measure is contained in $T\left\{n_{k}^{(3)}\right\}$.

(3) That the exceptional set of values of $t$ may be nonenumerable follows from the results of Erdös and Taylor or may be proved directly by constructing a function for which the exceptional set contains a residual $G_{\delta}$ and therefore is nonenumerable. For example let $\left\{t_{k}\right\}, k=1,2, \cdots$ be a sequence of points dense in the interval $1 \leqq t \leqq 2$ and define $\phi(x)$ by

$$
\begin{aligned}
\phi(x) & =\exp \left(i t_{k} x\right) \\
& =1
\end{aligned}
$$$$
4^{2 k} \pi \leqq x t_{k} \leqq 4^{2 k+1} \pi,
$$

all other $x$. 


\section{REFERENCES}

1. P. Erdös and S. J. Taylor, On the set of points of convergence of a lacunary trigonometric series and the equidistribution properties of related sequences, Proc. London Math. Soc. (2) vol. 7 (1957) pp. 598-615.

2. C. S. Herz, The Bohr spectrum of bounded functions, Bull. Amer. Math. Soc. vol. 62 (1955) p. 76.

3. H. Weyl, Über die Gleichverteilung von Zahlen $\bmod$ Eins, Math. Ann. vol. 77 (1915-1916) pp. 313-352.

Cambridge, England

\section{THE MULTIPLICATION PROBLEM FOR DIRICHLET SERIES}

\section{J. P. TULL}

E. Landau $[1, \S 214]$ has given a theorem on the multiplication of Dirichlet series to the effect that if $\alpha, \beta, \rho, \tau$, are real numbers with $\min (\rho, \tau)>\max (\alpha, \beta)$ and if $\sum a_{n} \xi_{n}^{-s}$ converges for $\sigma>\alpha$, absolutely for $\sigma>\rho, \sum b_{n} \xi_{n}^{-s}$ converges for $\sigma>\beta$, absoutely for $\sigma>\tau$, then the Dirichlet product of these two series converges for

$$
\sigma>\frac{o \tau-\alpha \beta}{\rho+\tau-\alpha-\beta} .
$$

(If $\min (\rho, \tau) \leqq \max (\alpha, \beta)$ then we have convergence for $\sigma>\max (\alpha, \beta)$.) H. Bohr $[2$, Theorem XIX] gave an example to show that in the case $\alpha=\beta=0, \rho=\tau=1$ the above conclusion cannot be improved.

In this paper we shall use a variation of Bohr's example to give, for each $\alpha, \beta, \rho, \tau$ with $\min (\rho, \tau)>\max (\alpha, \beta)$, two Dirichlet series whose product has abscissa of convergence exactly

$$
\frac{\rho \tau-\alpha \beta}{\rho+\tau-\alpha-\beta} \text {. }
$$

Thus we show that Landau's theorem is the best possible in all cases (the trivial cases being handled similarly).

Bohr [2, Theorem XVII] defines a certain Dirichlet series $\sum a_{m} m^{-s}$ as follows. Let $\left(\alpha_{n}\right),\left(t_{n}\right),\left(\beta_{n}\right),\left(\gamma_{n}\right)$ be sequences of positive integers such that for all $n \geqq 1$

Received by the editors September 16, 1957 and, in revised form, September 23, 1957. 